\title{
Nucleocytoplasmic transport in apoptosis
}

\author{
E Ferrando-May ${ }^{\star, 1}$ \\ ${ }^{1}$ Molecular Toxicology Group, Faculty of Biology, University of Konstanz, PO \\ Box X911, 78457 Konstanz, Germany \\ * Corresponding author: E Ferrando-May; Tel: + 497531 884054; \\ Fax: + 497531 884033; E-mail: elisa.may@uni-konstanz.de
}

Received 20.12.04; revised 14.2.05; accepted 15.2.05; published online 29.4.05 Edited by $L$ Greene

\begin{abstract}
The apoptotic demolition of the nucleus is accomplished by diverse proapoptotic factors, most of which are activated in the cytoplasm and gain access to the nucleoplasm during the cell death process. The nucleus is also the main target for genotoxic insult, a potent apoptotic trigger. Signals generated in the nucleus by DNA damage have to propagate to all cellular compartments to ensure the coordinated execution of cell demise. The nucleocytoplasmic shuttling of signalling and execution factors is thus an integral part of the apoptotic programme. Several proteins implicated in apoptotic cell death have been shown to migrate in and out of the nucleus following apoptosis induction. This review summarises the current knowledge on nucleocytoplasmic trafficking of apoptosis-relevant proteins. The effects of apoptosis induction on the nucleocytoplasmic transport machinery are also discussed. Finally, a potential role of nuclear transport as a critical control point of the apoptotic signal cascade is proposed.
\end{abstract}

Cell Death and Differentiation (2005) 12, 1263-1276.

doi:10.1038/sj.cdd.4401626; published online 29 April 2005

Keywords: nuclear permeability; nuclear pore; caspase; mitochondria; death domain

\footnotetext{
Abbreviations: AIF, apoptosis-inducing factor; APL, acute promyelocytic leukaemia; ATP, adenosine triphosphate; CARD, caspase-recruitment domain; DD, death domain; DDIA, DNA damage-induced apoptosis; DED, death effector domain; DEDD, death effector domain-bearing DNA-binding; DIO-1, death inducer obliterator 1; DISC, death-inducing signalling complex; FADD, Fas-associated death domain; FRET, fluorescence energy transfer; GAPDH, glyceraldehyde-3-phosphate dehydrogenase; GDP, guanosine diphosphate; GTP, guanosine triphosphate; LMB, leptomycin B; MEF, murine embryonic fibroblast; NES, nuclear export signal; NLS, nuclear localisation signal; NPC, nuclear pore complex; Nup, nucleoporin; PAR, poly-ADPribose; PML, promyelocytic leukaemia; $\mathrm{POD}, \mathrm{PML}$-oncogenic domain; RAR $\alpha$, retinoic acid receptor $\alpha$; TGF- $\beta$, transforming growth factor $\beta$; TNFR1, tumor necrosis factor receptor 1 ; TRADD, TNFR1-associated death domain; WGA, wheat germ agglutinin
}

\section{Introduction}

The separation of the nucleus and the cytoplasm is the defining feature of eukaryotic cells and is achieved by the nuclear envelope, a double-membrane system of highly selective permeability. Interchange of material between these two compartments occurs through dedicated transport channels perforating the nuclear envelope, the nuclear pore complexes (NPCs). These are elaborate supramolecular structures consisting of about 30 different proteins, most of which are termed nucleoporins (Nups). The composition and structure of the NPC have been analysed in detail by a combination of proteomics and electron microscopy approaches both in yeast and vertebrates, leading to a refined view of its molecular architecture. Essentially, the NPC is composed of three substructures of eight-fold rotational symmetry: the cytoplasmic fibrils, the central framework, and the nuclear basket (Figure 1). In the central framework, Nups form distinct subcomplexes which are arranged symmetrically with respect to the plane of the nuclear envelope and enclose the central pore channel. The peripheral structures are composed of Nups occurring uniquely either on the cytoplasmic or the nucleoplasmic side of the NPC.

The basic mechanisms underlying molecular transport between the nucleus and the cytoplasm have been characterised in detail and recent comprehensive reviews are available. In short, transport through the NPC may occur either by passive diffusion, according to the concentration gradient across the nuclear envelope, or actively, in a signaland energy-dependent manner. While passive transport is restricted to macromolecules smaller than $50-40 \mathrm{kDa}$, active transport can accommodate whole ribosomal subunits up to several million $\mathrm{Da}$ in size. This occurs in a complex process that includes the following steps: (1) recognition of the cargo's import/export signal (NLS/NES) by an import or export receptor (importins or exportins), (2) docking of the cargo/ receptor assembly at the NPC, (3) translocation across the NPC, (4) release of the transported protein, and (5) recycling of the receptors for the next round of transport (Figure 2).

The direction of transport across the nuclear envelope is determined by the small GTPase Ran, a predominantly nuclear protein, together with regulatory factors, such as the GTP-exchange factor RCC1 and the GTPase-activating protein RanGAP. RCC1 is a chromatin-binding protein and thus restricted to the nucleus. This ensures high nuclear levels of RanGTP. RanGAP is located at the cytoplasmic fibrils of the NPC and catalyses GTP hydrolysis by Ran. In this way, the cytoplasmic concentration of RanGTP is kept low. RanGTP affects the affinity of import and export receptors for their respective cargoes in opposite ways: it promotes the formation of export complexes and induces the dissociation of import complexes. Thus, exportins can bind NES-containing proteins only in the nucleus at high RanGTP levels, while 
importins can bind NLS-containing proteins only in the cytoplasm (Figure 2).

By determining the distribution of proteins between the nucleus and the cytoplasm, nucleocytoplasmic transport enables the spatial regulation of protein activity and may act as an integration point of diverse signalling systems. Accordingly, nuclear transport has been shown to control diverse biological processes, like cell division, motility, and the response to cytokines.

A recently emerging but still poorly explored link is that between nuclear transport and cell death by apoptosis. In fact, the number of known proteins whose function in apoptosis depends on regulated nuclear localisation is steadily increasing, supporting the notion that nucleocytoplasmic transport of signal transducers and execution factors may be a crucial and, in some systems, even essential aspect of cell death.

Several mechanisms exist by which cells regulate the nucleocytoplasmic distribution of proteins in response to diverse signalling cues. These are: (1) the masking/unmasking of nuclear transport signals by conformational changes in the cargo molecule as a consequence of protein oligomerisa- tion or post-translational modification, (2) the retention of cargo molecules in one compartment via tethering to an immobile cellular component, (3) the modulation of the activity of the mobile transport receptors, and (4) alterations of the transport channel, the NPC. Examples for each of these modes of regulation are found in cells undergoing apoptosis.

The purpose of this review is to summarise the current knowledge about the regulated nuclear localisation of proteins involved in apoptosis and to discuss the potential mechanisms of control and their impact on the transduction of apoptotic signals. Firstly, data concerning the effects of apoptosis induction on the integrity and selectivity of the nucleocytoplasmic transport machinery will be presented. The major apoptosis-related proteins known to move between the nucleus and the cytoplasm will then be overviewed and their function discussed from the perspective of regulated nuclear transport. Finally, the controversial issue of whether caspases, the major proteases acting in apoptosis, and caspaseactivated execution factors are actively imported into the nucleus will be addressed.

\section{Cracking the Barrier: Apoptotic Alterations of the Nuclear Transport System}

Regulation of the transport machinery provides a simple way to coordinately control the transport of multiple cargoes. Both the mobile components of the transport system, including the transport receptors as well as the members of the RanGTP. ase cycle, and the NPC are altered in apoptosis (Figure 3).

Evidence that cellular stress affects the soluble transport machinery initially came from yeast, where the steady-state localisation of the importin $\beta$-homolog Nmd5 was observed to change from mainly cytoplasmic to nuclear in response to osmotic shock. ${ }^{1}$ Similarly, starvation, heat shock, ethanol, and hydrogen peroxide were shown to interfere with the nuclear import of an NLS-containing reporter protein and to cause nucleocytoplasmic equilibration of the Ran-homolog Gsp1p. ${ }^{2}$

Figure 1 Schematic representation of the nuclear pore complex with its major substructures. A cross-section perpendicular to the plane of the nuclear envelope is shown

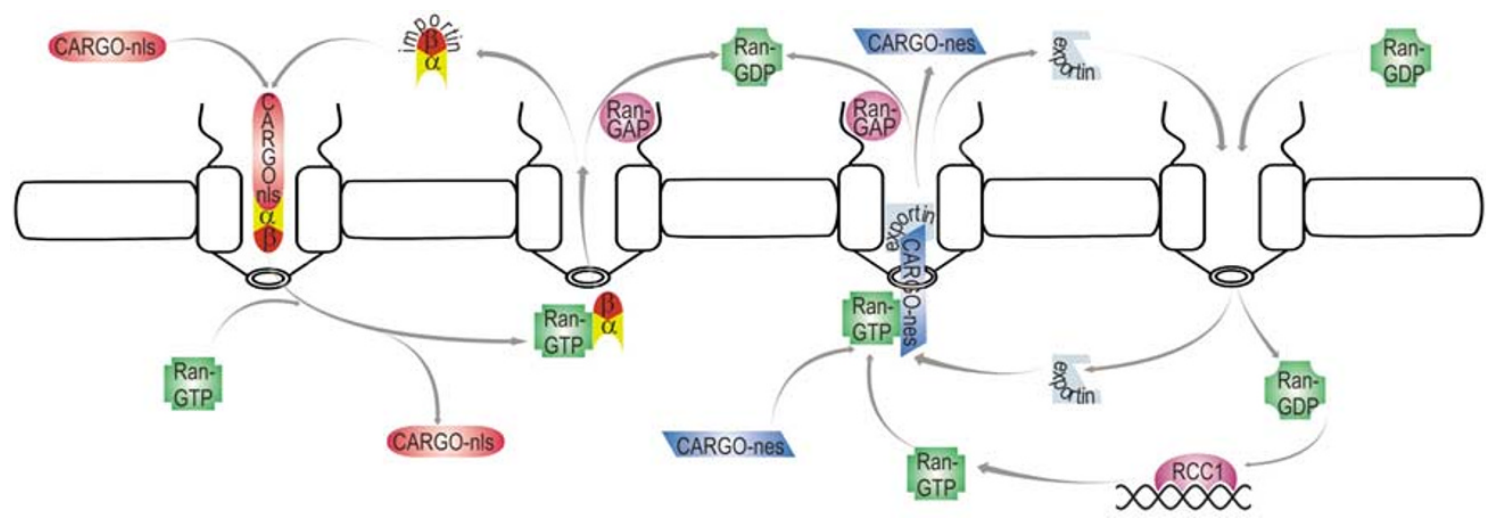

Figure 2 Active nucleocytoplasmic transport mediated by importins and exportins. Import substrates bearing an NLS are complexed by the importin $\alpha / \beta$ transport receptor heterodimer in the cytoplasm and translocated through the NPC. In the nucleus, RanGTP binds to the import substrate-receptor complex and induces cargo release. The importin-RanGTP complex is then recycled to the cytoplasm, where RanGTP is displaced from the complex and hydrolysed to RanGDP by RanGAP that is associated with the cytoplasmic filaments of the NPC. In the nucleus RanGTP promotes the formation of export complexes consisting of an export substrate carrying an NES, the export receptor (exportin), and RanGTP itself. After translocation to the cytoplasm, hydrolysis of RanGTP induces the release of free exportin and RanGDP. Both are reimported into the nucleus by independent mechanisms. Chromatin-bound RCC1 ensures that conversion of RanGDP to RanGTP occurs in the nucleus 
a

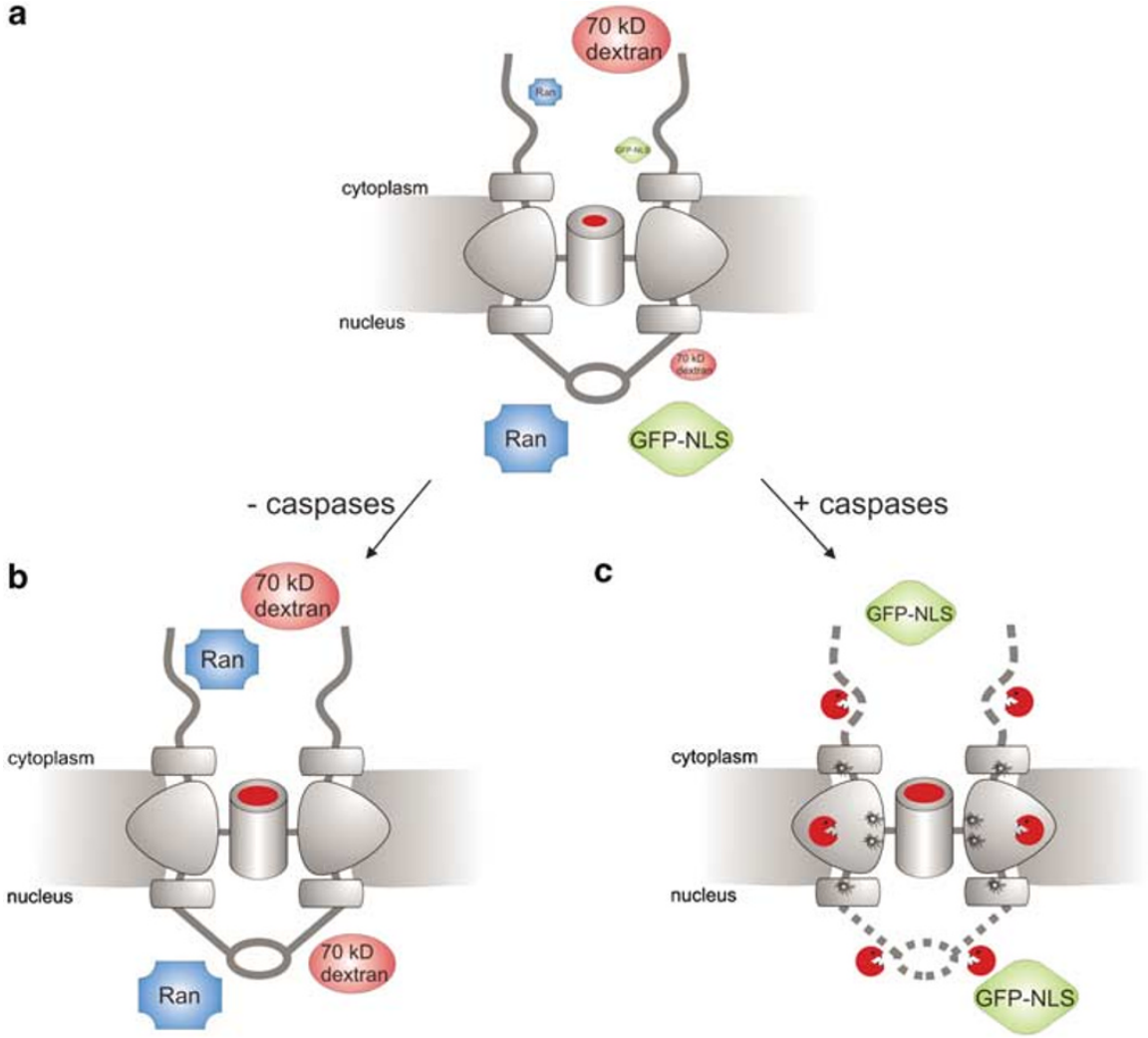

Figure 3 Alterations of the nucleocytoplasmic transport system in apoptosis. (a) The NPC as well as the distribution of the transport factor Ran, the size-exclusion marker for passive diffusion $70 \mathrm{kDa}$ dextran, and the import substrate GFP-NLS in nonapoptotic cells are indicated. (b) In the early phase of apoptosis as well as in the presence of caspase inhibitors, the size-exclusion limit of the nuclear envelope is increased, leading to the permeation of $70 \mathrm{kDa}$ dextran molecules into the nucleus. This is paralleled by the appearance of Ran in the cytoplasm. No data are available on the distribution of GFP-NLS at this stage. N.B. Also leakage of GFP-NLS and of a pentameric GFP fusion has been observed in cells with apparently intact NPCs. In this study, the effect was dependent on caspase-9 (see text). For reasons of clarity, these data were omitted from the figure. (c) Caspases attack the NPC in the execution phase of apoptosis. All the components of the peripheral structures are cleaved. In the central framework only two Nups located symmetrically with respect to the plane of the nuclear envelope are processed. Cleavage of the NPC coincides with the appearance of GFP-NLS in the cytoplasm

In mammalian cells, induction of apoptosis by staurosporine or actinomycin D was associated with the nucleocytoplasmic redistribution of the transport factors importin $\beta$, importin $\alpha$, and Ran. ${ }^{3}$ An increase in cytoplasmic Ran concentration was also observed after treatment with cisplatin, $\mathrm{H}_{2} \mathrm{O}_{2}$, and UV irradiation, suggesting that Ran's subcellular distribution may represent a general and early indicator of cellular stress and apoptosis. Since RanGAP, which is responsible for the hydrolysis of Ran-bound GTP, localises properly at the NPC and stays intact throughout apoptosis, ${ }^{3}$ it can be predicted that Ran entering the cytoplasm in the early phase of cell death will be converted to its GDP-bound state. This will result in a reduction of the cellular pool of RanGTP. In yeast and Xenopus oocytes, a depletion of RanGTP has been shown to inhibit NLS-dependent protein import most likely via the accumulation of the importin $\alpha$ transport receptor in the nucleus. ${ }^{4}$ Low RanGTP levels due to leakage of Ran from the nucleus may thus result in a decline of import activity also in cells undergoing apoptosis. This assumption is corroborated by a recent study showing collapse of the Ran gradient, importin $\alpha$ accumulation, and suppression of the classical import pathway in response to UV irradiation, oxidative, and heat stress. ${ }^{5}$
The apoptotic redistribution of Ran and other transport factors is an early feature of apoptosis which precedes caspase activation and is insensitive to pan-caspase inhibitors. $^{3,6}$ Analysis of the size exclusion limit of the nuclear envelope showed that, under these conditions, the permeability of the NPC to larger-sized dextrans $(70 \mathrm{kDa})$ increases, suggesting that the diameter of the NPC channel may be modulated early in apoptosis. ${ }^{3}$

Later in the apoptotic process, active caspases attack the nuclear transport system by cleaving components of the NPC. A recent comprehensive study investigated the fate of all known Nups in two different models of apoptosis. ${ }^{7}$ Out of 28 NPC components, only seven were found to be subject to caspase-mediated proteolysis. These are: Nup96 and Nup93 in the central framework; Nup153, Tpr, and Nup50 on the nucleoplasmic side, and RanBP2 and Nup214 at the cytoplasmic fibrils. The peripheral structures of the NPC are thus extensively degraded. In contrast, the central core, which constitutes the bulk mass of the NPC, is targeted at only two sites, Nup96 and Nup93. While elimination of the peripheral NPC components is unlikely to compromise the functional properties of the complex, cleavage of the framework nucleoporins Nup96 and Nup93 is predicted 
to impair the permeability barrier as well as active transport processes.

Interestingly, cytoplasmically oriented Nups seemed to be degraded prior to the nucleoplasmic Nups when apoptosis was triggered at the plasma membrane, while the opposite was observed when the apoptotic insult was directed to the nucleus by using a DNA-damaging agent. This suggests the existence of pathways leading to an early activation of caspases in the nucleus after genotoxic injury.

The time point of caspase-dependent proteolysis of the NPC has been correlated with the impaired ability of apoptotic cells to accumulate GFP-NLS into the nucleus. ${ }^{8}$ Whether this is exclusively due to leakage of GFP-NLS through the damaged NPC or due to a preceding, caspaseindependent decline in import activity or both has not been determined in detail. An increase in the cytoplasmic concentration of GFP-NLS during apoptosis was also observed in cells which lacked Nup proteolytic processing. ${ }^{9}$ In the latter study, the NPC was also shown to become permeable to a pentameric GFP molecule, supporting the above-mentioned idea that in apoptosis the nuclear permeability barrier might be altered or compromised even if the NPC is intact. These authors proposed that caspase-9 mediates the collapse of the permeability barrier by a yet undescribed mechanism which does not necessarily involve the cleavage of Nups. However, these results were challenged by another investigation showing that inhibition of caspase- 9 had no effect on the subcellular localisation of Ran in apoptotic cells. ${ }^{10}$

Despite some controversial data, these studies indicate that during apoptosis the nuclear transport machinery is regulated at least at two different levels: in the absence of active executioner caspases, that is, at the onset of apoptosis and/or under caspase-inhibiting conditions, the diameter of the NPC for passive diffusion is increased by as yet unknown mechanisms. This is concomitant with a redistribution of essential nuclear transport factors which may influence transport activity and/or selectivity. Similar changes of NPC permeability have been observed when cells transit from a quiescent to a proliferating state or in response to viral transformation. ${ }^{11}$ In the execution phase of apoptosis, the NPC is targeted by caspases which cleave a defined subset of Nups, thereby introducing irreversible changes in the architecture and functional properties of the transport channel.

Different transport mechanisms may thus apply for proapoptotic factors moving in and out of the nucleus at different stages and in different models of apoptosis. Proteins crossing the nuclear envelope in the absence of or prior to caspase activation will require active transport, depending on their molecular size. Here, the caspase-independent dilation of the NPC may shift the threshold of this requirement to higher values. In fact, early investigations indicated that small changes in NPC radius $(\sim 1 \mathrm{~nm})$ augment the half-time of nucleocytoplasmic equilibration for proteins larger than 40$50 \mathrm{kDa}$ by more than a 1000 -fold. ${ }^{12}$ On the other hand, proteins whose translocation requires active caspases or occurs downstream of caspase activation, are unlikely to encounter a functional barrier so that diffusion may contribute to their passage across the nuclear envelope.

\section{Hazardous Cargo: Mitochondrial Proteins Enter the Nucleus}

Mitochondria are known to harbour a whole battery of apoptogenic factors which are released upon selective permeabilisation of the outer mitochondrial membrane in the course of apoptosis and can act at different levels in the apoptotic signalling pathway. Some of them, like apoptosisinducing factor (AIF), the oxidoreductase WOX1, and endonuclease $G$ (EndoG) exert their action in the nucleus and have been shown to traverse the nuclear envelope during apoptosis (see Table 1).

\section{Apoptosis-inducing factor (AIF)}

Mammalian AIF is a mitochondrial flavoprotein of $\sim 67 \mathrm{kDa}$ with homology to bacterial oxidoreductases. ${ }^{13}$ Its primary sequence harbours two putative NLSs, the more C-terminal of which predominates and has been confirmed by deletion mutagenesis. ${ }^{14}$ When recombinant AIF is coincubated with purified nuclei or microinjected into the cytoplasm of healthy cells, it induces loss of DNA and peripheral chromatin condensation. These effects are independent of caspases as they are unaffected by caspase inhibitors, but require translocation of AIF through the NPC, as shown by microinjection experiments with wheat germ agglutinin (WGA), a lectin which binds O-glycosylated Nups thereby obstructing the NPC channel. Mitochondrio-nuclear translocation of AIF has been demonstrated by immunofluorescence and immunoelectron microscopy and, surprisingly, was not prevented by depletion of cellular ATP, ${ }^{15}$ which is known to block or at least interfere with active nuclear import. While the release of AIF from mitochondria may depend on caspases in some systems, but not in others, translocation into the nucleus does not require caspase activity, ${ }^{13,15}$ but is regulated by the heat shock protein hsp70. ${ }^{14}$ Downregulation of hsp70 by the antisense approach sensitises cells to AIF-mediated cell death. In cells triggered to undergo apoptosis, nuclear entry of AIF as well as AIF-associated chromatin alterations can be prevented by hsp70 overexpression.

\section{WOX1}

A second mitochondrial oxidoreductase which has been shown to migrate to the nucleus as a consequence of apoptosis induction is WOX1. Nuclear entry of WOX1 is mediated by a classical NLS sequence in the N-terminus of the protein. Mutation of this sequence efficiently reduced WOX1-dependent sensitisation to TNF-induced cell death. ${ }^{16}$ Phosphorylation of WOX1 at Tyr33 is essential for its cytotoxic effect, while caspase activity seems to be dispensable. ${ }^{16}$ Whether phosphorylation regulates WOX1 nuclear translocation remains to be determined.

\section{Endonuclease G}

Endonuclease $\mathrm{G}$ is a nonspecific DNA/RNA nuclease which is released from the intermembrane space of mitochondria during apoptosis, as shown both under cell-free conditions and in intact cells. ${ }^{17}$ The native nuclease occurs as a dimer of 
Table 1 Nucleocytoplasmic traffic of apoptosis-related proteins

\begin{tabular}{|c|c|c|c|c|}
\hline Protein & $\begin{array}{l}\text { Translocation direction } \\
\text { in apoptosis }\end{array}$ & Signals for active transport, regulation mechanisms & $\begin{array}{l}\text { Involvement of } \\
\text { caspases }\end{array}$ & References \\
\hline AIF & mito $\rightarrow$ nuc & NLS, cytoplasmic retention (hsp70) & casp. - indep. & 13,14 \\
\hline WOX-1 & mito $\rightarrow$ nuc & NLS & ND & 16 \\
\hline Endo G & mito $\rightarrow$ nuc & No obvious transport signals & ND & 17 \\
\hline ARTS & mito $\rightarrow$ nuc & ND & casp. - dep. & 91,100 \\
\hline DEDD & cyto $\rightarrow$ nuc & NLS, ubiquitination, cytoplasmic retention (cytokeratins) & casp. - dep. & 18,19 \\
\hline TRADD & cyto $\rightarrow$ nuc & NLS, NES, cytoplasmic retention (cytokeratins) & ND & 20,21 \\
\hline Apaf-1 & cyto $\rightarrow$ nuc & Mediated by nucling & ND & 30 \\
\hline DIO-1 & cyto $\rightarrow$ nuc & NLS, phosphorylation & ND & 39 \\
\hline GAPDH & cyto $\rightarrow$ nuc & NLS, NES, oligomerisation & ND & 41,42 \\
\hline MST1 & cyto $\rightarrow$ nuc & NLS, NES, phosphorylation & casp. - dep. & 87,101 \\
\hline PAK2 & cyto $\rightarrow$ nuc & NLS, NES & casp. - dep. & 88 \\
\hline Helicard & cyto $\rightarrow$ nuc & ND & casp. - dep. & 89 \\
\hline CAD/DFF40 & cyto $\rightarrow$ nuc & NLS & casp. - dep. & 90,102 \\
\hline HDAC4 & cyto $\rightarrow$ nuc & NLS, NES & casp. - dep. & 92 \\
\hline DAXX & nuc $\rightarrow$ cyto & NLS, NES, phosphorylation & ND & 35 \\
\hline PML & nuc $\rightarrow$ cyto & Induced by retroviral infection & ND & 38 \\
\hline Nur77/TR3 & nuc $\rightarrow$ mito & NLS, NES, phosphorylation & ND & 47,48 \\
\hline p53 & nuc $\rightarrow$ mito & NLS, NES, ubiquitination & ND & $54,58,60$ \\
\hline Histone H1.2 & nuc $\rightarrow$ mito & Active import by importin $\beta$ /importin 7 & casp. - indep. & 62,63 \\
\hline Caspase-2 & nuc $\rightarrow$ mito & NLS & casp. - dep. & 10,70 \\
\hline PAR & nuc $\rightarrow$ mito & ND & casp. - indep. & $\begin{array}{l}\text { Dawson, V } \\
\text { (unpublished) }\end{array}$ \\
\hline FADD & cyto $\leftrightarrow$ nuc & NLS, NES (exportin-5), phosphorylation & ND & 23,24 \\
\hline PEA-15 & cyto $\leftrightarrow$ nuc & NES & ND & 31,32 \\
\hline
\end{tabular}

about $50 \mathrm{kDa}$ and, once released, migrates to the nucleus where it can generate both high-molecular weight and oligonucleosomal-sized chromatin fragments. The primary sequence of Endo $\mathrm{G}$ does not harbour an obvious NLS (Meiss $\mathrm{G}$, pers. comm.) and the mechanism of nuclear entry has not yet been investigated in detail.

AIF as well as EndoG and WOX1 can exert their proapoptotic effect in the absence of caspase activation. In addition, AIF has been shown to localise to the nucleus of caspase-inhibited cells treated to undergo apoptosis. ${ }^{15}$ Therefore, caspase-dependent proteolysis of the NPC is most likely not required for the nuclear uptake of these proteins. NLS-deficient mutants of AIF and WOX1 are hampered in their apoptogenic function, suggesting that active import is required for their nuclear translocation upon release from the mitochondria. In untreated cells, mitochondrial sequestration of AIF, WOX1 and EndoG is most likely the major determinant of their nuclear exclusion, although binding to cytoplasmic tethering proteins like hsp70 or NLS masking by phosphorylation may contribute an additional level of regulation. The fact that ATP depletion has no effect on AIF nuclear translocation is difficult to reconcile with active nuclear import. However, unconventional, ATP-independent import pathways may play a role as already demonstrated for the MAP kinase ERK.

\section{Nucleocytoplasmic Trafficking of Death-fold Proteins}

Death-folds are signature structures found in proteins involved directly or indirectly in the activation of caspases.
They include the death domain (DD), the death effector domain (DED), and the caspase-recruitment domain (CARD). They all represent variations of a protein interaction module consisting of a compact bundle of six $\alpha$-helices. Death-folds were first identified in the intracellular portion of death receptors like the Fas/CD95 receptor and TNFR1 and were subsequently found to occur in combination with DEDs or CARDs in adapter molecules like FADD, TRADD, CRADD, RAIDD, and Apaf-1, involved in the relay of apoptotic signals within the cytoplasmic compartment.

\section{DEDD}

The discovery of DEDD, a death-effector-domain-bearing, DNA-binding protein that upon Fas/CD95 treatment translocated from the cytoplasm to the nucleus and potentiated apoptosis, provided the first example for a function of deathfolds within the nucleus. ${ }^{18}$ Recent studies on DEDD and its close homolog, DEDD2/FLAME-3, have demonstrated that the proapoptotic activity of these proteins is essentially dependent both on their translocation to the nucleus and the presence of a DED, since mutation of either DED or NLS abolishes apoptosis triggered by exogenous expression of DEDD or DEDD2. Ectopically expressed DEDD accumulates in the nucleus, where it colocalises with and specifically activates caspase- 6 .

Further studies revealed that endogenous DEDD is present both in the cytoplasm and in the nucleus. The interchange between these two pools is regulated by a complex mechanism. Cytoplasmic DEDD exists predominantly as a monoor diubiquitinated protein, the latter being associated with 
intermediate filaments. ${ }^{19}$ Nuclear DEDD is concentrated at nucleoli and is not ubiquitinated, indicating that ubiquitination may control the nucleocytoplasmic distribution of DEDD. Upon apoptosis induction, cytoplasmic and nuclear DEDD seem to undergo a reciprocal conformational change or a modification that influences the structure of an epitope lying within the DED. The DEDD-specific signal detected with a conformation-selective antibody in the nucleus of nonapoptotic cells vanishes upon induction of apoptosis. Concurrently, a DEDD-specific signal becomes visible in the cytoplasm at intermediate filaments using the same antibody. ${ }^{19}$ Under similar treatment conditions, cell fractionation and Western blot analysis showed that the bulk amount of DEDD in the nucleus does not diminish, but rather increases. ${ }^{18}$ Together, these data suggest that endogenous DEDD translocates to the nucleus upon induction of apoptosis and that this is associated with (a) structural alteration(s) of the protein.

Nuclear and cytoplasmic DEDD seem to promote apoptosis by distinct mechanisms: In the nucleus, DEDD interferes with the transcriptional activity of RNA polymerase I and transcription factor III (TFIIIC). In the cytosol, DEDD increments caspase-3 activation by recruiting and concentrating it to intermediate filaments. Interestingly, a mutant of DEDD which is defective in nuclear localisation is not ubiquitinated and interferes with DEDD-mediated caspase-3 activation in a dominant-negative manner, ${ }^{19}$ further supporting the existence of a close functional link between the nuclear and the cytoplasmic pools of DEDD. The nuclear translocation of DEDD as well as its apoptosis-induced conformational change and the subsequent formation of filamentous DEDD aggregates in the cytosol require caspase activity, as they are strongly reduced in the presence of caspase inhibitors.

Based on the available data, three mechanisms can be proposed for the regulation of DEDD's nucleocytoplasmic trafficking: first, ubiquitination may function as a signal for nuclear transport or influence the recognition of the existing NLSs, as has already been shown for the shuttling of transcription factors; second, tethering to cytoskeletal structures via the interaction with cytokeratin $\mathrm{K} 8 / 18$ may restrict DEDD in the cytoplasmic compartment; last but not least, DEDD's nuclear accumulation, which occurs downstream of caspase activation, may be facilitated by the proteolytic cleavage of the NPC.

The family of death-fold proteins with a conditional nuclear localisation includes at present four further members: TRADD, FADD, Apaf-1, and PEA-15.

\section{TRADD}

The adapter protein TNFR1-associated death domain (TRADD), a component of the TNFR1 signalling complex at the plasma membrane, contains nuclear export and import sequences that allow shuttling between the nucleus and the cytoplasm. ${ }^{20}$ Blocking nuclear export with leptomycin B (LMB) induces nuclear accumulation of TRADD, indicating that exportin-1, the export receptor which is specifically blocked by LMB, transports TRADD out of the nucleus. The NLS is located in the C-terminal DD of TRADD. A peptide corresponding to the DD localises exclusively to the nucleus and is a potent inducer of apoptosis. If the adjacent last 13 amino acids of TRADD are included in the expression construct, the encoded peptide becomes uniformly distributed between the cytoplasm and nucleus. ${ }^{20}$ Since the $\mathrm{C}$-terminal part of TRADD has been shown to bind to cytokeratin K18 and mediate TRADD localisation to intermediate filaments, ${ }^{21}$ it is likely that tethering to cytoskeletal structures might regulate TRADD nucleocytoplasmic distribution similarly to that of DEDD. Also resembling DEDD the nuclear and cytosolic pools of TRADD seem to promote apoptosis by distinct mechanisms, as shown by the different susceptibility to various inhibitors of apoptosis. In the nucleus, the DD of TRADD localises to PML-oncogenic domains (PODs), nuclear bodies enriched in the promyelocytic leukaemia protein (PML), a mediator of multiple apoptotic signals (e.g. Takahashi et al. ${ }^{22}$ ). Cell death elicited by the nuclear TRADD-DD requires PML and is not affected either by caspase inhibitors or by dominant-negative FADD, a potent antagonist of death-receptor signalling. On the contrary, the apoptogenic effect of wt-TRADD is significantly reduced by $z V A D$-fmk and dominant-negative FADD. ${ }^{20}$

In healthy cells TRADD localises both to the cytoplasm and to the perinuclear Golgi compartment, suggesting that nuclear export of TRADD predominates over import. Induction of apoptosis is likely to alter this equilibrium. Shifting the subcellular localisation of TRADD could provide a potential mechanism for switching from a caspase-dependent pathway mediated by cytosolic TRADD to a caspase-independent pathway involving nuclear TRADD. This hypothesis remains to be verified since nucleocytoplasmic translocation of TRADD upon TNFR1 stimulation or other apoptotic stimuli has not been demonstrated yet.

\section{FADD}

FADD is a bifunctional adapter molecule carrying a DD and a DED, which bridges the interactions between death receptors like TNFR1 and the Fas/CD95 receptor and the initiator caspases of the extrinsic pathway. Based on its function in death receptor signalling, FADD is expected to reside at the cell periphery, but recent work has shown that in adherent cells FADD is primarily present in the nucleus. ${ }^{23-25}$ In a lymphoid cell line population FADD localisation is not uniform, with approximately half of the cells having FADD in the nucleus. ${ }^{23}$ While the relative amounts of FADD present in the cytoplasm and the nucleus may depend on cell type and staining procedure ${ }^{25}$ its ability to translocate in and out of the nucleus is supported by the presence of NLS and NES signals in the $\mathrm{N}$-terminus of the protein ${ }^{23}$ and by its interaction with the transport receptor exportin-5. ${ }^{24}$ Nuclear localisation of FADD is regulated by phosphorylation at Ser194 in the C-terminus of the protein. Mutation of Ser194 or deletion of the DED, both reducing FADD/exportin-5 interaction, caused FADD to be uniformly distributed throughout the cell. ${ }^{24}$ In T-cells, Ser194 phosphorylation is mediated by a yet unknown cell-cycleregulated kinase. ${ }^{26}$ In adherent cells, detachment from the matrix induces export of FADD out of the nucleus, suggesting that Ser194 phosphorylation may be regulated by cell-matrix interactions. Whether FADD shuttles between the nucleus and the cytoplasm in response to an apoptotic stimulus remains to be investigated. 


\section{Apaf-1}

Apaf-1 was originally identified as a factor required for dATPtriggered activation of caspase- 3 in cytosolic extracts from normally growing cells. Via its N-terminal CARD motif, Apaf-1 contributes to the assembly of a supramolecular complex, termed apoptosome, which forms in the presence of cytochrome $C$ and dATP and serves as activation platform for caspase-9. ${ }^{27}$ Apaf-1 was reported to reside exclusively in the cytosol both in normal and apoptosing cells ${ }^{28}$ contrarily to its Caenorhabditis elegans homolog CED-4, which was shown to translocate from mitochondria to nuclear membranes in the early phase of embryonic cell death. ${ }^{29}$ Recent data, however, suggest that Apaf-1 might migrate to the nucleus also in mammalian cells during stress-induced apoptosis (e.g. Sakai et $a l^{30}$ ). Nuclear translocation of Apaf1 seems to be an early event preceding mitochondrial cytochrome $C$ release. Nucling, a protein of unknown function that is upregulated in cardiac muscle differentiation, has been proposed to act as a carrier for Apaf-1 nuclear uptake, since Nucling-/- MEFs do not show Apaf-1 relocalisation to the nucleus after UV irradiation. ${ }^{30}$ These cells are also resistant to UV-induced cell death, suggesting that Apaf-1 might exert a proapoptotic function in the nucleus, at least in some apoptosis model systems.

\section{PEA-15}

Pea-15 is a small DED-containing protein with a broad antiapoptotic action that was first identified as an abundant phosphoprotein in brain astrocytes. Pea-15 carries an Nterminal NES which directs the protein to the cytoplasm. Treatment with LMB leads to nuclear accumulation of Pea-15, indicating firstly that export is mediated by the LMB-sensitive export receptor exportin-1, and, secondly, that Pea-15 can shuttle between the nucleus and the cytoplasm. ${ }^{31}$ Nuclear entry of Pea-15 is slow, occurring most likely by diffusion. ${ }^{32}$ Similarly to TRADD and FADD, Pea-15 acts via different mechanisms in the cytoplasm and the nucleus: cytosolic Pea15 has been shown to bind via its DED to the death-inducing signalling complex (DISC) after TRAIL stimulation and to protect cells from apoptosis by preventing the activation of caspase- 8 at the DISC. Nuclear Pea- 15 has been proposed to bind and export the MAP-kinase ERK from the nucleus, thereby exerting an antiproliferative function. ${ }^{32}$

\section{Miscellaneous Apoptosis Regulators Moving in and out of the Nucleus \\ DAXX}

A factor involved in multiple apoptotic pathways whose subcellular localisation has been discussed controversially is Daxx (Fas Death Domain Associated Protein xx) (see e.g., Michaelson ${ }^{33}$ ). Originally discovered in a yeast two-hybrid screen aimed at identifying proteins that interact with the cytoplasmic tail of the Fas/CD95 receptor, Daxx was then reported to be an exclusively nuclear protein localised to PODs and to act as a transcriptional corepressor by its interaction with several DNA-associated proteins. Other studies showed that Daxx mediates the interaction between the Fas/CD95 receptor and the JNK kinase kinase Ask-1 in the cytoplasm, leading to kinase activation and potentiation of Fas/CD95-induced cell death.

Daxx bears two putative NLSs ${ }^{34}$ and one NES, ${ }^{35}$ predicting that it may shuttle between the nucleus and the cytosol. Recently, a mechanism for the regulation of Daxx's nucleocytoplasmic translocation has been proposed that reconciles some of the conflicting data about Daxx localisation and function. ${ }^{35}$ According to this model, Daxx exits the nucleus under conditions of cellular stress, as shown in glucosedeprived and $\mathrm{H}_{2} \mathrm{O}_{2}$-treated cells. The export of Daxx is triggered by phosphorylation at Ser667, which induces a conformational change that unmasks the NES, thereby promoting interaction with the export receptor exportin-1. ${ }^{35}$ In the cytoplasm, Daxx activates Ask-1, which in turn triggers JNK-1. Active JNK-1 was shown to indirectly increment Daxx phosphorylation and export, suggesting the existence of a positive feedback loop in the regulation of Daxx nucleocytoplasmic shuttling.

Regulated nuclear localisation of Daxx may explain to some extent the contradictory features of this protein, which has been reported to have both pro- and antiapoptotic functions depending on the cellular context. ${ }^{33}$ Change of Daxx's localisation from nuclear to cytoplasmic may contribute to switching its activity as an essential regulator of transcription required for cell survival to that of a sensitising factor lowering the threshold for death in cells exposed to environmental challenges.

\section{PML}

Acute promyelocytic leukaemia (APL), a specific subtype of acute myeloid leukaemia, is the consequence of a chromosomal translocation that fuses the tumor suppressor gene $P M L$ to the retinoic acid receptor alpha gene $(R A R \alpha)$ (reviewed in Zelent et $a l^{36}$ ). Expression of the fusion protein in promyelocytic blasts interferes with the normal subcellular localisation of $\mathrm{PML}$, preventing its concentration in nuclear subdomains termed PODs or nuclear domains 10 (ND10). PML has been implicated in growth suppression, apoptosis, and replicative senescence. Formation of PODs in the nucleus appears crucial for PML's role in modulating these diverse physiological responses, although the exact function of these subnuclear structures is still elusive (see Takahashi et al. ${ }^{22}$ and references therein). The PML protein exists as seven major isoforms generated by alternative splicing, but not all of them localise to the nucleus. Recently, a cytoplasmic PML isoform (cPML) has been shown to function as an essential modulator of signalling cascades triggered by the cytokine transforming growth factor $\beta$ (TGF- $\beta),{ }^{37}$ indicating that PML may contribute to distinct signalling pathways depending on its subcellular localisation. PML has also been shown to move from the nucleus to the cytoplasm as a consequence of retroviral infection, ${ }^{38}$ raising the possibility that PML's multiple functions may be regulated at the level of nuclear transport.

\section{DIO-1}

A further mediator of apoptosis, whose activity requires nuclear translocation, is death inducer-obliterator-1, DIO-1, 
a protein expressed in the limb interdigital membranes during development. In healthy cells, DIO-1 is located in the cytoplasm. When apoptosis is triggered either by IL-3 starvation or c-myc induction or simply by DIO-1 overexpression, DIO-1 translocates to the nucleus and induces via an as yet unknown mechanism the upregulation of procaspase levels and apoptotic cell death. The DIO-1 sequence carries a canonical bipartite NLS. Deletion of the NLS prevents DIO-1 nuclear entry and impairs its ability to induce apoptosis. The mechanisms responsible for triggering DIO-1 nuclear uptake during apoptosis are unclear so far, but phosphorylation might play a role, since differences in the phosphorylation status of DIO-1 between the cytosolic and the nuclear population of $\mathrm{DIO}-1$ have been described. ${ }^{39}$

\section{GAPDH}

Glyceraldehyde-3-phosphate dehydrogenase (GAPDH) is a well-known housekeeping protein and a key enzyme of glycolysis. In vivo it is present as a tetramer of identical $37 \mathrm{kDa}$ subunits, as a dimer or as the $37 \mathrm{kDa}$ monomer. The glycolytic function is restricted to the tetramer. ${ }^{40}$

During the last 10 years, novel activities of GAPDH have emerged, including that of a mediator of apoptotic cell demise. $^{40}$ The proapoptotic effects of GAPDH were linked to its ability to shuttle between the cytoplasm and the nucleus. The C-terminus of the protein harbours a sequence resembling a bipartite NLS. Recent mutational analysis, however, uncovered the presence of an unconventional NES in the same region, which mediates interaction with the export receptor exportin-1. Inhibition of exportin-1-GAPDH interaction by LMB induced nuclear accumulation of GAPDH. ${ }^{41}$ Interestingly, nuclear entry of GAPDH seems to depend on the oligomerisation state of the protein: inhibitors of GAPDH which induce the dissociation of the active tetrameric form into free dimers inhibited GAPDH nuclear translocation and reduced apoptosis. ${ }^{42}$

GAPDH has been implicated in the control of apoptosis particularly in neuronal cells. Treatment with antisense GAPDH constructs prevented neuronal apoptosis and GAPDH nuclear translocation. Furthermore, GAPDH has been shown to selectively bind in vitro to proteins implicated in neuronal disease like $\beta$-amyloid precursor protein, huntingtin, and other proteins involved in the pathogenesis of triplet repeat genetic disorders (reviewed in Mazzola and Sirover ${ }^{43}$ ). This has fostered the hypothesis that GAPDH may bind and promote the nuclear accumulation of mutated neuronal proteins, thereby contributing to the pathogenesis of neurodegenerative diseases.

\section{The Nucleus Strikes Back: The Release of Apoptogenic Nuclear Proteins}

In 1994, two parallel studies concerning the relevance of the nucleus for the transduction of apoptotic signals showed that enucleated cells (cytoplasts) can undergo morphological changes characteristic of programmed cell death and concluded that 'the presence of a nucleus.... is not required to transmit apoptotic signals'. ${ }^{44,45}$ Meanwhile, the focus of interest has shifted from the execution to the commitment phase of apoptosis, leading to a rediscovery of the cell's organelles, including the nucleus, as damage sensors and initiators of the apoptotic cascade.

Being the primary site of genotoxic damage, the nucleus obviously plays a fundamental role in DNA-damage induced apoptosis (DDIA). The pathways involved in DDIA have been reviewed recently. ${ }^{46}$ Interestingly, some of the factors mediating DNA damage are proteins that directly transmit apoptotic signals by translocating out of the nucleus. This is observed not only in DDIA, but also in several other paradigms of apoptosis.

\section{Nur77/TR3}

The first example of such a nuclear messenger of apoptosis was the transcription factor Nur77/TR3, a member of the orphan steroid receptor family. Nur77/TR3 was originally characterised as an immediate-early gene expressed in response to mitogenic signals and was later found to be rapidly upregulated in thymocytes undergoing apoptosis in response to T-cell receptor (TCR) engagement. Nur77/TR3 antisense constructs or a dominant-negative mutant protect from TCR-triggered cell death, while constitutive expression of Nur77/TR3 induces a massive apoptotic response. Nur77/ TR3 has been shown to mediate apoptosis by translocating from the nucleus to the mitochondria where it induces the release of cytochrome $C .^{47}$ Blocking Nur77/TR3 nuclearmitochondrial translocation, which occurs in several types of cancer cells, prevented apoptosis induced by different stimuli (e.g. Li et al. ${ }^{47}$ and Wilson et al. ${ }^{48}$ ).

Recently, Nur77/TR3 has been shown to bind to Bcl-2 once released from the nucleus. This interaction is required for Nur77/TR3 mitochondrial targeting and triggers a conformational change in the $\mathrm{Bcl}-2$ molecule, which exposes the proapoptotic $\mathrm{BH} 3$ domain, thereby converting $\mathrm{Bcl}-2$ from an anti- to a proapoptotic factor. ${ }^{49}$

Nur77/TR3 carries two NLSs and three NESs. ${ }^{50}$ In PC12 phaeochromocytoma cells, Nur77/TR3 is actively exported out of the nucleus in response to treatment with nerve growth factor. This involves phosphorylation of Ser105. ${ }^{50}$ Further, Nur77/TR3 has been shown to heterodimerise and to translocate out of the nucleus together with retinoid $\mathrm{X}$ receptor $\alpha$, which subsequently has been identified as the carrier for Nur77/TR3 nuclear export in gastric cancer cells. In these cells, export was triggered by 9-cis retinoic acid and resulted in mitochondrial association of Nur77/TR3 and apoptotic cell death. Experiments with the exportin-1 inhibitor LMB suggest that Nur77/TR3 is actively exported from the nucleus also in cells undergoing apoptosis after DNA damage. ${ }^{47,48}$ In cerebellar granule neurons exposed to excess glutamate nuclear exit of Nur77/ TR3 involved the MAP-kinase ERK, indicating that phosphorylation may play a role in regulating Nur77/TR3 localisation also in apoptotic cells. This is further supported by the observation made by Lin et al. $^{49}$ that the electrophoretic mobility of Nur77/TR3 changes when it translocates to mitochondria. 


\section{p53}

In the nucleus p53 controls the cellular response to DNA damage via the transcriptional induction or repression of a wide range of genes. This may result either in cell cycle arrest, DNA repair, or apoptosis. Recent in vivo experiments have demonstrated that the proapoptotic function of p53 is largely dependent on its transcriptional activity in the nucleus. ${ }^{51,52}$ Several studies, however, have reported that p53 may also mediate apoptosis independently from gene transactivation (reviewed in Chipuk and Green ${ }^{53}$ ). The first clue that this may involve an extranuclear activity of p53 came from a study showing that $\mathrm{p} 53$ can rapidly translocate to the mitochondrial membrane shortly after the induction of DNA damage. ${ }^{54}$ Further studies have corroborated this initial finding, showing that direct targeting of p53 to the mitochondria initiates a rapid wave of apoptosis in the thymus and the spleen of $\gamma$-irradiated mice, which precedes the p53-dependent transcriptional response. $^{55}$

At the mitochondria p53 interacts with members of the Bcl-2 family of regulators of mitochondrial permeability (reviewed in Chipuk and Green ${ }^{56}$ ). Direct complex formation of p53 with $\mathrm{Bcl}-\mathrm{XI}$ and $\mathrm{Bcl}-2$ has been shown to promote mitochondrial cytochrome $C$ release. This involves the liberation of proapoptotic Bax or Bak from their inhibitory association with $\mathrm{Bcl}-\mathrm{Xl} / \mathrm{Bcl}-2$. A further study reported a direct interaction of p53 with Bak, leading to Bak oligomerisation and mitochondrial permeabilisation. ${ }^{57}$ Independently of their relative contribution to the transcription-independent proapoptotic function of $\mathrm{p53}$, these interactions with $\mathrm{Bcl}-2$ family members require a fast and efficient accumulation of p53 in the cytoplasm following an apoptotic trigger. The nucleocytoplasmic transport of p53 is mediated by three distinct NLSs in the C-terminus of the protein and by two NES located in the $\mathrm{N}$ - and C-terminal regions. ${ }^{58,59}$

The link between p53 nuclear export and its propapototic function at the mitochondria has not been extensively explored yet. It is known that phosphorylation inhibits and monoubiquitination promotes nuclear export of $p 53 .^{60}$ Recent work has shown that p53 can be mono- or polyubiquitinated depending on the cellular level of its cognate E3-ubiquitin ligase, Mdm2, and that monoubiquitinated p53 is readily exported out of the nucleus while the polyubiquitinated form is not. ${ }^{61}$ In light of these data, the following hypothetical scenario can be drawn: under stressfree conditions, when the Mdm2 level is low, monoubiquitination and thus nuclear export of p53 will prevail. When a proapoptotic insult hits the cell cytoplasmic p53 present at a low steady-state level could be rapidly targeted to mitochondria and ensue the described first rapid wave of cell death. Later in the apoptotic process p53's transcriptional activity comes into play, leading to an increased expression of the Mdm2 gene, which is under the control of a p53responsive promoter. At this point, phosphorylation of p53 will become predominant, resulting in the accumulation of p53 in the nucleus and the shut-off of the N-terminal NES. The apoptotic pathway will then be primarily influenced by the transcription-dependent activity of p53, while polyubiquitination will ensure degradation of the protein in the late downregulatory phase.

\section{Histone H1.2}

Looking for factors in the thymic cytosol of $\gamma$-irradiated mice that could trigger cytochrome $C$ release from purified mitochondria, Konishi et al. ${ }^{62}$ determined that the most potent activity was due to histone $\mathrm{H} 1.2$, a conclusion confirmed using recombinant $\mathrm{H} 1.2$. The action of $\mathrm{H} 1.2$ at the mitochondria seems to involve Bak oligomerisation since mitochondria from Bak-deficient mice were relatively resistant to the effect of H1.2. Following DNA damage, H1.2 translocates from the nucleus to the cytoplasm. Whether this occurs by active export is still debated since no canonical NES has been identified in the $\mathrm{H} 1.2$ sequence, but the translocation process is sensitive to LMB. ${ }^{62}$ In healthy cells histone $\mathrm{H} 1$ is imported very efficiently into the nucleus by the importin $\beta$ /importin 7 receptor heterodimer. ${ }^{63}$ An alternative mechanism for the appearance of $\mathrm{H} 1.2$ in the cytoplasm of irradiated cells could be a decrease in the rate of active import due to an impaired activity of the nucleocytoplasmic transport machinery (see above). The caspase-dependent proteolysis of the NPC most likely does not play a role in the cytoplasmic accumulation of H1.2 which occurs also in Apaf-1-deficient cells lacking caspase-3/-9 activity.

Interestingly, the release of $\mathrm{H} 1.2$ from the nucleus is essentially dependent on p53, since it is absent in the cytosolic fraction isolated from the thymus of $\mathrm{p} 53^{-/-}$mice. The connection between p53 and the nuclear release of $\mathrm{H} 1.2$ is still unclear, but may reflect the role of p53 in global chromatin relaxation after DNA damage, as recently demonstrated in UV-treated cells. In fact, histone $\mathrm{H} 1$ was shown to be released from chromatin as a consequence of large-scale chromatin rearrangements induced by DNA damage. ${ }^{64}$

\section{Caspase-2}

Caspase-2 is the only caspase known so far that localises constitutively to the nucleus. ${ }^{10,65}$ Nuclear import is mediated by two classical NLSs in the prodomain of the precursor enzyme, the most $\mathrm{C}$-terminal of which binds to the importin $\alpha / \beta$ heterodimer. Ablation of caspase-2 expression inhibits cell death induced by trophic factor deprivation and DNA damage in different cell types, supporting an apical role of this caspase in stress-induced apoptosis. ${ }^{66}$ Moreover, the mechanism of caspase-2 activation resembles that of the initiator caspase-8 and -9 , since it does not involve proteolytic processing of the precursor. $^{67}$

Activation of caspase-2 requires the formation of a large protein complex termed PIDDosome, which lacks both Apaf-1 and cytochrome $C$ but contains the death domain protein PIDD and the adapter RAIDD. ${ }^{68,69}$ Interestingly, overexpressed caspase-2 localises to dot- and filament like structures in the nucleus probably reflecting the formation of PIDDosomes. How this process, which requires both PIDD and RAIDD, ${ }^{69}$ is initiated in the nuclear compartment is the subject of current investigation. While endogenous RAIDD is present in the nuclear compartment, PIDD has been reported to localise to the cytoplasm and to translocate to the nucleus upon induction of apoptosis, triggering PIDDosome formation and caspase-2 activation (Tschopp J, pers. comm.). These data, which still await confirmation, provide the first example 
of a caspase activation pathway regulated at the level of nuclear transport.

Nuclear transport most likely influences also the execution of apoptosis downstream of caspase-2 activation, which involves the release of cytochrome $C$ and Smac/Diablo from the mitochondria. In vitro experiments with purified caspase-2 and isolated mitochondria indicate that this caspase can engage directly the mitochondrial apoptotic pathway by permeabilising the outer mitochondrial membrane. ${ }^{70}$ It can thus be predicted that release of processed caspase- 2 from the nucleus during apoptosis will suffice to trigger cytochrome $C$ release and the execution of the apoptotic degradation programme similarly to Nur77/TR3, p53, and histone H1.2. Whether caspase-2 translocates out of the nucleus in the early stage of cell death is still unknown. Microinjection experiments showed that a caspase-2-GFP fusion protein is retained in the nucleus until late apoptosis and that LMB could not prevent death induced by caspase-2 overexpression, arguing against a selective release of this protease from the nuclear compartment. ${ }^{10}$ Whether this reflects the behaviour of the endogenous protein in cells undergoing apoptosis remains to be determined.

\section{Poly-ADP-ribose (PAR)}

Poly-ADP-ribose polymerase-1 (PARP-1) is an abundant nuclear enzyme which binds to DNA and stimulates DNA repair. PARP-1 is activated by DNA breaks and catalyses the transfer of ADP-ribose moieties from $\mathrm{NAD}^{+}$to a variety of acceptor proteins including PARP-1 itself. This leads to the formation of complex protein-bound poly-ADP-ribose polymers with chain lengths of up to 200 units and multiple branching points. ${ }^{71}$

Besides its activity as a mediator of DNA repair, PARP-1 has also been implicated in the control of cell death by apoptosis. In particular, neuronal apoptosis after ischemiareperfusion or glutamate excitotoxicity has been shown to require PARP-1 activation. In these model systems, PARP-1 is required for the mitochondrial release of AIF, indicating that a PARP-1-dependent nuclear signal is generated early after damage and propagates to mitochondria. ${ }^{72}$ This signal is assumed to be the PAR polymer itself since a PAR-specific signal is detected in the cytoplasm of neurons challenged with a high dose of glutamate. More significantly, synthetic purified PAR can release AIF from isolated mitochondria (Dawson $\mathrm{V}$, pers. comm.). If confirmed, these data may define the PAR polymer as a novel type of signalling molecule in the nuclearmitochondrial crosstalk in apoptosis.

\section{The Open Question: Are Caspases (and Caspase-activated Executors) Actively Imported into the Nucleus?}

The nuclear localisation of either precursor or mature caspases is still a matter of debate. In early immunohistochemical investigations, caspase-3 and caspase-1 immunoreactivity was detected in the nuclei of a subpopulation of cells in neuroblastic tumors with favourable prognosis ${ }^{73}$ and nuclear caspase-3 staining was observed in healthy tissue of different origin, as for example testis and colon. ${ }^{74}$ However, these studies did not differentiate between the zymogens and the processed active caspases. Subsequently, subcellular fractionation and affinity labelling experiments identified multiple mature caspases in the nuclear fraction of cells induced to undergo apoptosis. ${ }^{75-77}$ These were: caspase$3,{ }^{75-77}$ caspase- $6,{ }^{76}$ caspase- $2,{ }^{75}$ and caspase-8. ${ }^{75,78} \mathrm{Ma}-$ ture caspase- 9 and -7 were reported to translocate to the nucleus in some studies, but not in others. ${ }^{75}$ Among the caspase zymogens, only procaspase-2 has been definitely identified as a bona fide nuclear protein. ${ }^{65}$ Nuclear localisation of procaspase- 8 and procaspase- 9 was proposed, but not confirmed. ${ }^{75}$

The controversy about the nuclear localisation of caspases and procaspases most likely reflects differences between cell types and apoptotic triggers as well as problems inherent to the methods employed, first of all the isolation of subcellular fractions and the labelling with caspase-specific probes targeted to the active centre of the enzyme. The isolation of nuclear fractions from apoptotic cells bears a higher risk of contamination with non-nuclear components as compared to healthy cells, due to the compromised stability of intracellular membranes, for example, by ROS production during the apoptotic process (see e.g., the discrepancy between the results on caspase-2 localisation obtained by cell fractionation and by immunofluorescence ${ }^{10}$ ). Moreover, it was reported that nuclei lose more than $90 \%$ of their protein content when isolated by conventional procedures. ${ }^{79}$ Finally, the affinity labelling of caspases bears several pitfalls which have been reviewed recently. ${ }^{80}$ A more promising approach to determine caspase distribution and activation at the subcellular level is in vivo imaging. Several papers have reported the use of fluorescence energy transfer (FRET) to monitor caspase activity in living cells. Recently, caspase-3 activity in the nucleus has been measured with a FRET reporter carrying an NLS, which allowed to detect caspase-3 activation prior to the appearance of morphological signs of apoptosis in TNF $\alpha$ treated HeLa cells. ${ }^{81}$ A further study, in which the distribution of unprocessed caspases was investigated in different cell lines by means of GFP fusions, showed a remarkable variability in the degree of nuclear localisation of different caspases. $^{82}$

Until now, a classical NLS has been positively identified only in the prodomain of caspase- $2 .{ }^{65}$ Procaspase- 1 was also reported to bear an NLS; these results, however, were based solely on subcellular fractionation experiments. ${ }^{83}$ The absence of canonical transport sequences in the caspases' primary structures however does not rule out the possibility that they may enter the nucleus by alternative, NLSindependent active transport pathways, as reported, for example, for $\beta$-catenin or ERK2. ${ }^{84,85}$ In fact, Kamada et al. recently proposed that active caspase- 3 is translocated into the nucleus of apoptotic cells by association with a yet unknown substrate molecule. ${ }^{86}$

Independently from the mechanism in question, the issue of active nuclear uptake of caspases must be discussed in the context of the apoptosis-induced alterations of the nucleocytoplasmic barrier which were presented above. If in the initial stage of cell death the size exclusion limit of the NPC augments in such a way as to accommodate molecules of at 
least $70 \mathrm{kDa}$, caspases which in their active tetrameric form have a molecular weight of about $60 \mathrm{kDa}$ may not be efficiently retained outside of the nucleus. A requirement for active import of caspases seems questionable if we consider further that the NPC is an early target of caspase activity and that Nups crucially involved in cargo translocation and NPC selectivity are efficiently cleaved by caspases, independently of whether the apoptotic signal cascade is triggered inside or outside of the nucleus. Cleavage of these Nups is predicted to destroy the selective barrier function of the NPC. ${ }^{7}$

The same considerations apply for the nuclear import of apoptotic regulators which are activated downstream of caspases, or as a result of caspase activity. Examples for apoptotic executors which are activated by caspase cleavage and subsequently translocate to the nucleus are the protein kinases MST1 and PAK2. Caspase-mediated proteolysis removes the regulatory kinase domain which harbours the signals for nuclear export. ${ }^{87,88}$ In the case of MST1, nuclear localisation of the catalytic domain was shown to be necessary for its proapoptotic function. ${ }^{87}$ Also, factors directly involved in chromatin fragmentation are activated by caspases like Helicard and CAD/DFF40. Processing of Helicard, a CARD-containing helicase, by caspases generates a C-terminal fragment with helicase activity which translocates to the nucleus and accelerates DNA degradation. ${ }^{89}$ CAD/DFF40, the major DNAse responsible for oligonucleosomal DNA fragmentation, was initially proposed to move to the nucleus upon removal of the inhibitory subunit ICAD/DFF45 by caspases, although this model has been challenged by reports showing that ICAD is a nuclear protein, at least in some cell types (reviewed in Nagata et $\mathrm{al}^{90}$ ). Further proapoptotic factors translocating to the nucleus as a consequence of caspase activity are the mitochondrial protein ARTS ${ }^{91}$ and the $\mathrm{N}$-terminal fragment of histone deacetylase $4 .^{92}$

\section{Nuclear Transport as a Potential Decision Point in Apoptosis}

If caspases and caspase-dependent apoptotic executors may not need active import to access the nucleus, why does inhibition of import block or at least significantly delay the onset of cell death ${ }^{13,93,94}$ ? First of all, the requirement for active transport has mostly been inferred from experiments using WGA as a tool to inhibit translocation through the NPC. By binding to the heavily O-glycosylated Nups, WGA obstructs the NPC, but might also sterically shield Nups from caspase attack, thereby protecting the nucleocytoplasmic barrier. In fact, the concomitant microinjection of WGA and active caspase- 3 results in exclusion of this caspase from the nucleus and delayed cell death. ${ }^{93,94}$ Secondly, active nucleocytoplasmic transport may play a more significant role in caspase-independent modes of cell death, like those which involve mitochondrial AIF release. Last but not least, active nucleocytoplasmic transport may be required upstream of caspase activation. In fact, partial protection from apoptosis has been obtained also by inhibiting active transport with GTP- $\gamma$ S, and with excess amounts of NTF2/p10 or importin $\alpha{ }^{93}$
An intriguing hypothesis deserving further investigation is that nucleocytoplasmic transport may be part of a commitment step upstream of caspase activation and controlled by Bcl-2. Immunohistochemical and electron microscopy studies have convincingly demonstrated that $\mathrm{Bcl}-2$ localises not only to the mitochondria and the endoplasmic reticulum, but also to the nuclear envelope and here possibly to the NPC (see e.g., Lithgow et al. ${ }^{95}$ ). Moreover, Bcl-2 has been shown to inhibit the nuclear translocation of granzymes, of p53, and of the $\mathrm{NF} \kappa \mathrm{B}$ subunits $\mathrm{p} 50$ and $\mathrm{p} 65$, as well as to promote nuclear accumulation of reduced glutathione, thus supporting a function of $\mathrm{Bcl}-2$ in the regulation of nucleocytoplasmic exchange. On the other hand, recent studies have demonstrated that cells in which the mitochondrial pathway of caspase activation is abrogated by deletion of Apaf- 1 and caspase $-9^{96}$ or which lack both initiator caspases, caspase- 9 and caspase-2, are still capable to undergo stress-induced apoptosis unless Bcl-2 is overexpressed. ${ }^{97,98}$ Therefore, the existence of $\mathrm{Bcl}-2$-regulated component(s) of the intrinsic cell death pathway acting independently of the apoptosome and of caspase-2 has been proposed. The protective effect of $\mathrm{Bcl}-2$ on caspase-9- and caspase-2-independent cell death might be explained by the maintenance of mitochondrial respiratory function and normal energy metabolism or by inhibition of a still unknown initiator caspase. A third scenario might include the control of (a) nucleocytoplasmic translocation event(s) required for triggering executioner caspases independently of caspase-2 and the apoptosome. Clarifying the contribution of nuclear transport to Bcl-2's antiapoptotic function remains a challenging question for future investigations.

\section{Concluding Remarks}

Since the discovery of the first mammalian import receptors 10 years ago impressive progress has been made in the elucidation of the mechanisms that control the transport of molecules in and out of the nucleus. This has also been the time period in which apoptosis research has developed into a major field of cell biology and some of its crucial pathways have been unraveled. We are now in a position to apply our detailed knowledge of the processes responsible for the nuclear targeting or exclusion of proteins to understand how apoptosis may be regulated at the level of nucleocytoplasmic transport. There is plenty of evidence in the literature, that both the nuclear uptake and the nuclear release of apoptotic factors play important roles in the execution as well as in the initiation of the apoptotic program. The pathways involved in the transport of apoptotic factors are mostly unknown, but cleavage of crucial components of the NPC by caspases is likely to mark the switch from tight and controlled separation of nucleus and cytoplasm to a much less restricted exchange of material between the two compartments. Only very few factors known to cross the nuclear envelope in apoptosis have been characterised with respect to the role of caspases in the translocation process (see Table 1). This needs to be addressed in the future. A further point of interest concerns the identity of the transport receptors involved in translocation as well as their dependence on the RanGTPase system and of the cellular ATP level since the apoptotic cell might engage 
unconventional transport pathways to ensure correct protein targeting under compromised physiological conditions.

If, on the one hand, nucleocytoplasmic exchange of molecules may be essential for the execution of the apoptotic programme, global inhibition of nuclear transport on the other hand may represent a powerful apoptotic trigger, as highlighted by a recent study on the proapoptotic inhibitor of nuclear import CC3. ${ }^{99}$ How impaired nuclear transport may link to the core apoptotic machinery is a further question to explore in this emerging field at the intersection of apoptosis and nuclear transport research.

\section{Note added in proof}

While this article was under review, Bender et al. have shown that nuclear TRADD may induce apoptosis by a mechanism requiring caspase- 9 activity (Bender et al., Cell Death and Differentiation, 2005, epub ahead of print).

\section{Acknowledgements}

I would like to thank the members of the May Lab, V Cordes, and U Moll for helpful discussions, and S Beneke, J Diefenbach, A Deol, and A Bürkle for critical reading of the manuscript. I apologise for any contributions that could not be cited because of strict space limitations. Supplemental references are available at http://gutenberg.biologie.uni-konstanz.de/efm/ efm.html. This work was supported by the Deutsche Forschungsgemeinschaft (FOR 324/2-1/2-1).

\section{References}

1. Ferrigno P, Posas F, Koepp D, Saito $H$ and Silver PA (1998) Regulated nucleo/cytoplasmic exchange of HOG1 MAPK requires the importin beta homologs NMD5 and XPO1. EMBO J. 17: 5606-5614

2. Stochaj U, Rassadi R and Chiu J (2000) Stress-mediated inhibition of the classical nuclear protein import pathway and nuclear accumulation of the small GTPase Gsp1p. FASEB J. 14: 2130-2132

3. Ferrando-May E, Cordes V, Biller I, Görlich D, Mirkovic $J$ and Nicotera $P$ (2001) Caspases mediate nucleoporin cleavage but not early redistribution of transport factors and mRNA in apoptosis. Cell Death Differ. 8: 495-505

4. Schwoebel ED, Ho TH and Moore MS (2002) The mechanism of inhibition of Ran-dependent nuclear transport by cellular ATP depletion. J. Cell Biol. 157: 963-974

5. Miyamoto Y, Saiwaki T, Yamashita J, Yasuda Y, Kotera I, Shibata S, Shigeta M, Hiraoka Y, Haraguchi T and Yoneda Y (2004) Cellular stresses induce the nuclear accumulation of importin alpha and cause a conventional nuclear import block. J. Cell Biol. 165: 617-623

6. Kodiha M, Chu A, Matusiewicz N and Stochaj U (2004) Multiple mechanisms promote the inhibition of classical nuclear import upon exposure to severe oxidative stress. Cell Death Differ. 11: 862-874

7. Patre M, Tabbert A, Hermann D, Walczak H, Rackwitz HR, Cordes VC and Ferrando-May E (2005) Caspases target only two architectural components within the core structure of the nuclear pore complex (submitted)

8. Kihlmark M, Rustum C, Eriksson C, Beckman M, Iverfeldt $\mathrm{K}$ and Hallberg $\mathrm{E}$ (2004) Correlation between nucleocytoplasmic transport and caspase-3dependent dismantling of nuclear pores during apoptosis. Exp. Cell Res. 293: 346-356

9. Faleiro $L$ and Lazebnik $Y(2000)$ Caspases disrupt the nuclear-cytoplasmic barrier. J. Cell Biol. 151: 951-959

10. Paroni G, Henderson C, Schneider C and Brancolini C (2002) Caspase-2 can trigger cytochrome $C$ release and apoptosis from the nucleus. J. Biol. Chem. 277: 15147-15161
11. Feldherr CM and Akin D (1993) Regulation of nuclear transport in proliferating and quiescent cells. Exp. Cell Res. 205: 179-186

12. Paine PL, Moore LC and Horowitz SB (1975) Nuclear envelope permeability. Nature 254: 109-114

13. Susin SA, Lorenzo HK, Zamzami N, Marzo I, Snow BE, Brothers GM, Mangion J, Jacotot E, Costantini P, Loeffler M, Larochette N, Goodlett DR, Aebersold R, Siderovski DP, Penninger JM and Kroemer G (1999) Molecular characterization of mitochondrial apoptosis-inducing factor. Nature 397: 441-446

14. Gurbuxani S, Schmitt E, Cande C, Parcellier A, Hammann A, Daugas E, Kouranti I, Spahr C, Pance A, Kroemer G and Garrido C (2003) Heat shock protein 70 binding inhibits the nuclear import of apoptosis-inducing factor. Oncogene 22: 6669-6678

15. Daugas E, Susin SA, Zamzami N, Ferri KF, Irinopoulou T, Larochette N, Prevost MC, Leber B, Andrews D, Penninger J and Kroemer G (2000) Mitochondrio-nuclear translocation of AIF in apoptosis and necrosis. FASEB J. $14: 729-739$

16. Chang NS, Doherty J and Ensign A (2003) JNK1 physically interacts with WW domain-containing oxidoreductase (WOX1) and inhibits WOX1-mediated apoptosis. J. Biol. Chem. 278: 9195-9202

17. Li LY, Luo $X$ and Wang $X$ (2001) Endonuclease $G$ is an apoptotic DNase when released from mitochondria. Nature 412: 95-99

18. Stegh AH, Schickling O, Ehret A, Scaffidi C, Peterhansel C, Hofmann TG, Grummt I, Krammer PH and Peter ME (1998) DEDD, a novel death effector domain-containing protein, targeted to the nucleolus. EMBO J. 17: $5974-5986$

19. Lee JC, Schickling O, Stegh AH, Oshima RG, Dinsdale D, Cohen GM and Peter ME (2002) DEDD regulates degradation of intermediate filaments during apoptosis. J. Cell Biol. 158: 1051-1066

20. Morgan M, Thorburn J, Pandolfi PP and Thorburn A (2002) Nuclear and cytoplasmic shuttling of TRADD induces apoptosis via different mechanisms. J. Cell Biol. 157: 975-984

21. Inada H, Izawa I, Nishizawa M, Fujita E, Kiyono T, Takahashi T, Momoi T and Inagaki M (2001) Keratin attenuates tumor necrosis factor-induced cytotoxicity through association with TRADD. J. Cell Biol. 155: 415-426

22. Takahashi Y, Lallemand-Breitenbach V, Zhu J and de The H (2004) PML nuclear bodies and apoptosis. Oncogene 23: 2819-2824

23. Gomez-Angelats M and Cidlowski JA (2003) Molecular evidence for the nuclear localization of FADD. Cell Death Differ. 10: 791-797

24. Screaton RA, Kiessling S, Sansom OJ, Millar CB, Maddison K, Bird A, Clarke $A R$ and Frisch SM (2003) Fas-associated death domain protein interacts with methyl-CpG binding domain protein 4: a potential link between genome surveillance and apoptosis. Proc. Natl. Acad. Sci. USA 100: 5211-5216

25. Frisch $S$ (2004) Nuclear localization of FADD protein. Cell Death Differ. 11: 1361-1362

26. Scaffidi C, Volkland J, Blomberg I, Hoffmann I, Krammer PH and Peter ME (2000) Phosphorylation of FADD/MORT1 at serine 194 and association with a 70-kDa cell cycle-regulated protein kinase. J. Immunol. 164: 1236-1242

27. Zou H, Li Y, Liu X and Wang X (1999) An APAF-1 cytochrome c multimeric complex is a functional apoptosome that activates procaspase-9. J. Biol. Chem. 274: 11549-11556

28. Hausmann G, O'Reilly LA, van Driel R, Beaumont JG, Strasser A, Adams JM and Huang DC (2000) Pro-apoptotic apoptosis protease-activating factor 1 (Apaf-1) has a cytoplasmic localization distinct from bcl-2 or bcl-x(L). J. Cell Biol. 149: 623-634

29. Chen F, Hersh BM, Conradt B, Zhou Z, Riemer D, Gruenbaum Y and Horvitz HR (2000) Translocation of $C$. elegans CED-4 to nuclear membranes during programmed cell death. Science 287: 1485-1489

30. Sakai T, Liu L, Teng X, Mukai-Sakai R, Shimada H, Kaji R, Mitani T, Matsumoto M, Toida K, Ishimura K, Shishido Y, Mak TW and Fukui K (2004) Nucling recruits Apaf-1/pro-caspase- 9 complex for the induction of stressinduced apoptosis. J. Biol. Chem. 279: 41131-41140

31. Formstecher E, Ramos JW, Fauquet M, Calderwood DA, Hsieh JC, Canton B, Nguyen XT, Barnier JV, Camonis J, Ginsberg MH and Chneiweiss H (2001) PEA-15 mediates cytoplasmic sequestration of ERK MAP kinase. Dev. Cell 1: 239-250

32. Whitehurst AW, Robinson FL, Moore MS and Cobb MH (2004) The death effector domain protein PEA-15 prevents nuclear entry of ERK2 by inhibiting required interactions. J. Biol. Chem. 279: 12840-12847 
33. Michaelson JS (2000) The Daxx enigma. Apoptosis 5: 217-220

34. Pluta AF, Earnshaw WC and Goldberg IG (1998) Interphase-specific association of intrinsic centromere protein CENP-C with HDaxx, a death domain-binding protein implicated in Fas-mediated cell death. J. Cell Sci. 111 2029-2041

35. Song JJ and Lee YJ (2004) Tryptophan 621 and serine 667 residues of Daxx regulate its nuclear export during glucose deprivation. J. Biol. Chem. 279: 30573-30578

36. Zelent A, Guidez F, Melnick A, Waxman S and Licht JD (2001) Translocations of the RARalpha gene in acute promyelocytic leukemia. Oncogene 20: 7186-7203

37. Lin HK, Bergmann S and Pandolfi PP (2004) Cytoplasmic PML function in TGF-beta signalling. Nature 431: 205-211

38. Turelli P, Doucas V, Craig E, Mangeat B, Klages N, Evans R, Kalpana G and Trono D (2001) Cytoplasmic recruitment of INI1 and PML on incoming HIV preintegration complexes: interference with early steps of viral replication. Mol. Cell 7: 1245-1254

39. Garcia-Domingo D, Ramirez D, Gonzalez de Buitrago G and Martinez AC (2003) Death inducer-obliterator 1 triggers apoptosis after nuclear translocation and caspase upregulation. Mol. Cell. Biol. 23: 3216-3225

40. Sirover MA (1999) New insights into an old protein: the functional diversity of mammalian glyceraldehyde-3-phosphate dehydrogenase. Biochim. Biophys. Acta 1432: 159-184

41. Brown VM, Krynetski EY, Krynetskaia NF, Grieger D, Mukatira ST, Murti KG, Slaughter CA, Park HW and Evans WE (2004) A novel CRM1-mediated nuclear export signal governs nuclear accumulation of glyceraldehyde-3phosphate dehydrogenase following genotoxic stress. J. Biol. Chem. 279: 5984-5992

42. Carlile GW, Chalmers-Redman RM, Tatton NA, Pong A, Borden KE and Tatton WG (2000) Reduced apoptosis after nerve growth factor and serum withdrawal: conversion of tetrameric glyceraldehyde-3-phosphate dehydrogenase to a dimer. Mol. Pharmacol. 57: 2-12

43. Mazzola JL and Sirover MA (2002) Alteration of intracellular structure and function of glyceraldehyde-3-phosphate dehydrogenase: a common phenotype of neurodegenerative disorders? Neurotoxicology 23: 603-609

44. Schulze-Osthoff K, Walczak H, Droge W and Krammer PH (1994) Cell nucleus and DNA fragmentation are not required for apoptosis. J. Cell Biol. 127: 15-20

45. Jacobson MD, Burne JF and Raff MC (1994) Programmed cell death and Bcl2 protection in the absence of a nucleus. EMBO J. 13: 1899-1910

46. Norbury CJ and Zhivotovsky B (2004) DNA damage-induced apoptosis. Oncogene 23: 2797-2808

47. Li H, Kolluri SK, Gu J, Dawson MI, Cao X, Hobbs PD, Lin B, Chen G, Lu J, Lin F, Xie Z, Fontana JA, Reed JC and Zhang X (2000) Cytochrome $c$ release and apoptosis induced by mitochondrial targeting of nuclear orphan receptor TR3. Science 289: 1159-1164

48. Wilson AJ, Arango D, Mariadason JM, Heerdt BG and Augenlicht LH (2003) TR3/Nur77 in colon cancer cell apoptosis. Cancer Res. 63: 5401-5407

49. Lin B, Kolluri SK, Lin F, Liu W, Han YH, Cao X, Dawson MI, Reed JC and Zhang XK (2004) Conversion of Bcl-2 from protector to killer by interaction with nuclear orphan receptor Nur77/TR3. Cell 116: 527-540

50. Katagiri Y, Takeda K, Yu ZX, Ferrans VJ, Ozato K and Guroff G (2000) Modulation of retinoid signalling through NGF-induced nuclear export of NGFI-B. Nat. Cell Biol. 2: 435-440

51. Chao C, Saito S, Kang J, Anderson CW, Appella E and Xu Y (2000) p53 transcriptional activity is essential for p53-dependent apoptosis following DNA damage. EMBO J. 19: 4967-4975

52. Jimenez GS, Nister M, Stommel JM, Beeche M, Barcarse EA, Zhang XQ, O'Gorman S and Wahl GM (2000) A transactivation-deficient mouse mode provides insights into Trp53 regulation and function. Nat. Genet. 26: 37-43

53. Chipuk JE and Green DR (2003) p53's believe it or not: lessons on transcription-independent death. J. Clin. Immunol. 23: 355-361

54. Marchenko ND, Zaika A and Moll UM (2000) Death signal-induced localization of p53 protein to mitochondria. A potential role in apoptotic signaling. J. Biol. Chem. 275: 16202-16212

55. Erster S, Mihara M, Kim RH, Petrenko O and Moll UM (2004) In vivo mitochondrial p53 translocation triggers a rapid first wave of cell death in response to DNA damage that can precede p53 target gene activation. Mol. Cell. Biol. 24: 6728-6741
56. Chipuk JE and Green DR (2004) Cytoplasmic p53: bax and forward. Cell Cycle 3: 429-431

57. Leu Jl, Dumont P, Hafey M, Murphy ME and George DL (2004) Mitochondrial p53 activates Bak and causes disruption of a Bak-Mcl1 complex. Nat. Cell Biol. 6: 443-450

58. Liang SH and Clarke MF (2001) Regulation of p53 localization. Eur. J. Biochem. 268: 2779-2783

59. Stommel JM, Marchenko ND, Jimenez GS, Moll UM, Hope TJ and Wahl GM (1999) A leucine-rich nuclear export signal in the p53 tetramerization domain: regulation of subcellular localization and p53 activity by NES masking. EMBO J. 18: 1660-1672

60. Gottifredi V and Prives C (2001) Molecular biology. Getting p53 out of the nucleus. Science 292: 1851-1852

61. Li M, Brooks CL, Wu-Baer F, Chen D, Baer R and Gu W (2003) Mono- versus polyubiquitination: differential control of p53 fate by Mdm2. Science 302: 1972-1975

62. Konishi A, Shimizu S, Hirota J, Takao T, Fan Y, Matsuoka Y, Zhang L, Yoneda Y, Fujii Y, Skoultchi Al and Tsujimoto Y (2003) Involvement of histone H1.2 in apoptosis induced by DNA double-strand breaks. Cell 114: 673-688

63. Jakel S, Albig W, Kutay U, Bischoff FR, Schwamborn K, Doenecke D and Gorlich D (1999) The importin beta/importin 7 heterodimer is a functional nuclear import receptor for histone H1. EMBO J. 18: 2411-2423

64. Rogakou EP, Boon C, Redon C and Bonner WM (1999) Megabase chromatin domains involved in DNA double-strand breaks in vivo. J. Cell Biol. 146: 905-916

65. Colussi PA, Harvey NL and Kumar S (1998) Prodomain-dependent nuclear localization of the caspase-2 (Nedd2) precursor. J. Biol. Chem. 273: 24535-24542

66. Troy CM and Shelanski ML (2003) Caspase-2 redux. Cell Death Differ. 10: 101-107

67. Baliga BC, Read SH and Kumar S (2004) The biochemical mechanism of caspase-2 activation. Cell Death Differ. 11: 1234-1241

68. Read SH, Baliga BC, Ekert PG, Vaux DL and Kumar S (2002) A novel Apaf-1-independent putative caspase-2 activation complex. J. Cell Biol. 159: 739-745

69. Tinel A and Tschopp J (2004) The PIDDosome, a protein complex implicated in activation of caspase-2 in response to genotoxic stress. Science 304: 843-846

70. Robertson JD, Gogvadze V, Kropotov A, Vakifahmetoglu H, Zhivotovsky B and Orrenius S (2004) Processed caspase-2 can induce mitochondriamediated apoptosis independently of its enzymatic activity. EMBO Rep. 5: 643-648

71. Bürkle A (2001) Physiology and pathophysiology of poly(ADP-ribosyl)ation. BioEssays 23: 795-806

72. Hong SJ, Dawson TM and Dawson VL (2004) Nuclear and mitochondrial conversations in cell death: PARP-1 and AIF signaling. Trends Pharmacol. Sci. 25: 259-264

73. Nakagawara A, Nakamura $Y$, Ikeda $H$, Hiwasa T, Kuida K, Su MS, Zhao H, Cnaan A and Sakiyama S (1997) High levels of expression and nuclear localization of interleukin-1 beta converting enzyme (ICE) and CPP32 in favorable human neuroblastomas. Cancer Res. 57: 4578-4584

74. Krajewska M, Wang H-G, Krajewski S, Zapata JM, Shabaik A, Gascoyne R and Reed JC (1997) Immunohistochemical analysis of in vivo patterns of expression of CPP32 (Caspase-3), a cell death protease. Cancer Res. 57: $1605-1613$

75. Zhivotovsky B, Samali A, Gahm A and Orrenius S (1999) Caspases: their intracellular localization and translocation during apoptosis. Cell Death Differ. 6: 644-651

76. Martins LM, Kottke T, Mesner PW, Basi GS, Sinha S, Frigon Jr N, Tatar E, Tung JS, Bryant K, Takahashi A, Svingen PA, Madden BJ, McCormick DJ, Earnshaw WC and Kaufmann SH (1997) Activation of multiple interleukin-1beta converting enzyme homologues in cytosol and nuclei of HL-60 cells during etoposide-induced apoptosis. J. Biol. Chem. 272: 7421-7430

77. Mandal M, Adam L and Kumar R (1999) Redistribution of activated caspase-3 to the nucleus during butyric acid-induced apoptosis. Biochem. Biophys. Res. Commun. 260: 775-780

78. Benchoua A, Couriaud C, Guegan C, Tartier L, Couvert P, Friocourt G, Chelly $\mathrm{J}$, Menissier-de Murcia J and Onteniente B (2002) Active caspase-8 
translocates into the nucleus of apoptotic cells to inactivate poly(ADP-ribose) polymerase-2. J. Biol. Chem. 277: 34217-34222

79. Paine PL, Austerberry CF, Desjarlais LJ and Horowitz SB (1983) Protein loss during nuclear isolation. J. Cell Biol. 97: 1240-1242

80. Pozarowski P, Huang X, Halicka DH, Lee B, Johnson G and Darzynkiewicz Z (2003) Interactions of fluorochrome-labeled caspase inhibitors with apoptotic cells: a caution in data interpretation. Cytometry 55A: 50-60

81. Takemoto K, Nagai T, Miyawaki A and Miura M (2003) Spatio-temporal activation of caspase revealed by indicator that is insensitive to environmental effects. J. Cell Biol. 160: 235-243

82. Shikama Y, U M, Miyashita T and Yamada M (2001) Comprehensive studies on subcellular localizations and cell death-inducing activities of eight GFPtagged apoptosis-related caspases. Exp. Cell Res. 264: 315-325

83. Mao P-L, Jiang Y, Wee BY and Porter AG (1998) Activation of caspase-1 in the nucleus requires nuclear translocation of pro-caspase- 1 mediated by its prodomain. J. Biol. Chem. 273: 23621-23624

84. Fagotto F, Gluck U and Gumbiner BM (1998) Nuclear localization signalindependent and importin/karyopherin-independent nuclear import of beta-catenin. Curr. Biol. 8: 181-190

85. Whitehurst AW, Wilsbacher JL, You Y, Luby-Phelps K, Moore MS and Cobb MH (2002) ERK2 enters the nucleus by a carrier-independent mechanism. Proc. Natl. Acad. Sci. USA 99: 7496-74501

86. Kamada S, Kikkawa U, Tsujimoto $Y$ and Hunter $T$ (2005) Nuclear translocation of caspase- 3 is dependent on its proteolytic activation and recognition of a substrate-like protein(s). J. Biol. Chem. 280: 857-860

87. Ura S, Masuyama N, Graves JD and Gotoh Y (2001) Caspase cleavage of MST1 promotes nuclear translocation and chromatin condensation. Proc Natl. Acad. Sci. USA 98: 10148-10153

88. Jakobi R, McCarthy CC, Koeppel MA and Stringer DK (2003) Caspaseactivated PAK-2 is regulated by subcellular targeting and proteasomal degradation. J. Biol. Chem. 278: 38675-38685

89. Kovacsovics M, Martinon F, Micheau O, Bodmer JL, Hofmann K and Tschopp $\mathrm{J}$ (2002) Overexpression of Helicard, a CARD-containing helicase cleaved during apoptosis, accelerates DNA degradation. Curr. Biol. 12: 838-843

90. Nagata S, Nagase H, Kawane K, Mukae N and Fukuyama H (2003) Degradation of chromosomal DNA during apoptosis. Cell Death Differ. 10: 108-116

91. Larisch S, Yi Y, Lotan R, Kerner H, Eimerl S, Tony Parks W, Gottfried Y, Birkey Reffey S, de Caestecker MP, Danielpour D, Book-Melamed N, Timberg
R, Duckett CS, Lechleider RJ, Steller H, Orly J, Kim SJ and Roberts AB (2000) A novel mitochondrial septin-like protein, ARTS, mediates apoptosis dependent on its P-loop motif. Nat. Cell Biol. 2: 915-921

92. Paroni G, Mizzau M, Henderson C, Del Sal G, Schneider C and Brancolini C (2004) Caspase-dependent regulation of histone deacetylase 4 nuclearcytoplasmic shuttling promotes apoptosis. Mol. Biol. Cell. 15: 2804-2818

93. Yasuhara N, Eguchi Y, Tachibana T, Imamoto N, Yoneda $Y$ and Tsujimoto $Y$ (1997) Essential role of active nuclear transport in apoptosis. Genes Cells 2 55-64

94. Tautorat V, Haenisch C, Wendel A and Meergans T (2005) Role of active nuclear transport in caspase-3 translocation and chromatin destruction (submitted)

95. Lithgow T, van Driel R, Bertram JF and Strasser A (1994) The protein product of the oncogene bcl-2 is a component of the nuclear envelope, the endoplasmic reticulum, and the outer mitochondrial membrane. Cell Growth Differ. 5: 411-417

96. Marsden VS, O'Connor L, O'Reilly LA, Silke J, Metcalf D, Ekert PG, Huang DC, Cecconi F, Kuida K, Tomaselli KJ, Roy S, Nicholson DW, Vaux DL, Bouillet P, Adams JM and Strasser A (2002) Apoptosis initiated by Bcl-2regulated caspase activation independently of the cytochrome c/Apaf-1/ caspase-9 apoptosome. Nature 419: 634-637

97. Marsden VS, Ekert PG, Van Delft M, Vaux DL, Adams JM and Strasser A (2004) Bcl-2-regulated apoptosis and cytochrome c release can occur independently of both caspase-2 and caspase-9. J. Cell Biol. 165: 775-780

98. Ekert PG, Read SH, Silke J, Marsden VS, Kaufmann H, Hawkins CJ, Gerl R, Kumar S and Vaux DL (2004) Apaf-1 and caspase-9 accelerate apoptosis, but do not determine whether factor-deprived or drug-treated cells die. J. Cell Biol. 165: 835-842

99. King FW and Shtivelman E (2004) Inhibition of nuclear import by the proapoptotic protein CC3. Mol. Cell. Biol. 24: 7091-7101

100. Gottfried Y, Rotem A, Lotan R, Steller H and Larisch S (2004) The mitochondrial ARTS protein promotes apoptosis through targeting XIAP. EMBO J. 23: 1627-1635

101. Lee KK and Yonehara S (2002) Phosphorylation and dimerization regulate nucleocytoplasmic shuttling of mammalian STE20-like kinase (MST). J. Biol. Chem. 277: 12351-12358

102. Enari M, Sakahira H, Yokoyama H, Okawa K, Iwamatsu A and Nagata S (1998) A caspase-activated DNase that degrades DNA during apoptosis, and its inhibitor ICAD. Nature 391: 43-50 